\title{
Pronóstico del mieloma indolente
}

\author{
Prognosis of smoldering (asymptomatic) multiple myeloma
}

Kyle RA col. N Engl J Med 2007;357(18):1799-809

\section{Objetivo}

Evaluar al momento del diagnostico de mieloma indolente (MI) los factores de riesgo que se asocian a la progresión a mieloma múltiple (MM) o amiloidosis.

\section{Diseño}

Estudio retrospectivo de una cohorte de pacientes con MI.

\section{Lugar}

Mayo Clinic Cancer Center, Rochester, Minessota, EEUU.

\section{Pacientes}

Sobre una cohorte de 3549 pacientes con MM evaluados entre 1970 y 1995, se analizó retrospectivamente a los 276 con diagnóstico de $\mathrm{Ml}$, definido por la presencia de un componente monoclonal de IgG ó IgA mayor a 3g/dL y/o infiltración plas- mocitaria mayor a $10 \%$ en la médula ósea (MO) sin compromiso de otros órganos.

\section{Factores pronósticos evaluados}

Sexo, nivel de albúmina y hemoglobina, magnitud del componente monoclonal (más de $4 \mathrm{~g} / \mathrm{dL}$ ) tipo de cadenas livianas y pesadas involucradas, reducción de las inmunoglobulinas no comprometidas, expresión de Ciclina D1, proporción de células plasmáticas y tipo de infiltración plasmocitaria en la MO.

\section{Resultados Principales}

Durante un periodo de seguimiento de 2131 personas/año acumulativo hubo 163 pacientes con progresión de la enfermedad. Se establecieron tres grupos pronósticos según el porcentaje de infiltración plasmocitaria de la MO y los niveles de la paraproteina monoclonal. Ver tabla 1.

Tabla 1: probabilidad de progresión de los pacientes con mieloma indolente a mieloma múltiple o amiloidosis según el grado de infiltración plasmocitaria en la médula ósea y los niveles de paraproteína monoclonal.

\begin{tabular}{|c|c|c|c|c|c|c|c|}
\hline \multirow[t]{2}{*}{ Grupo } & \multirow[t]{2}{*}{ pts } & \multicolumn{2}{|c|}{ Definición del grupo de riesgo } & \multicolumn{2}{|c|}{ Cantidad de casos con } & \multicolumn{2}{|c|}{ Progresión a mieloma activo 0 amiloidosis $(p<0,001)$} \\
\hline & & $\begin{array}{l}\text { Grado de infiltración } \\
\text { plasmocitaria en la médula ósea }\end{array}$ & $\begin{array}{l}\text { Niveles de } \\
\text { paraproteína } \\
\text { monoclonal }\end{array}$ & Mieloma múltiple & Amiloidosis & $\begin{array}{l}\text { Probabilidad } \\
\text { acumulativa a } 15 \text { años }\end{array}$ & $\begin{array}{l}\text { Tiempo medio de la } \\
\text { progresión }\end{array}$ \\
\hline 1 & 106 & $10 \%$ ó más & $3 \mathrm{~g} / \mathrm{dL}$ ó más & 75 & 5 & $87 \%$ & 2 años \\
\hline 2 & 143 & $10 \%$ ó más & Menos de $3 \mathrm{~g} / \mathrm{dL}$ & 72 & 3 & $70 \%$ & 8 años \\
\hline 3 & 27 & Menos de $10 \%$ & $3 \mathrm{~g} / \mathrm{dL}$ más & 10 & 2 & $39 \%$ & 19 años \\
\hline
\end{tabular}

Otras variables de significancia pronóstica fueron la presencia de paraproteina IgA $(p=0,004)$ y la de cadenas livianas en la orina $(p=0,04)$; una infiltración plasmocitaria mayor a $20 \%$ en la MO $(p<0,001)$, la reducción del nivel de inmunoglobulinas no involucradas $(p=0,001)$ y el patrón de la infiltración en la MO $(p<0,001)$.

\section{Conclusiones}

El riesgo de progresión desde Ml a enfermedad sintomática esta relacionado al porcentaje de infiltración plasmocitaria en la $\mathrm{MO}$ y al nivel del componente monoclonal al momento del diagnostico.

Palabras claves: mieloma múltiple, mieloma múltiple indolente, pronóstico, evolución clínica.

Key words: Multiple Myeloma, asymptomatic multiple myeloma, prognosis of Key words: Multiple Myeloma,
Smoldering, clinical evolution.

Smoldering, clinical evolution.
Fuente de financiamiento: National Cancer Institute.

\section{Comentario}

El tiempo de seguimiento de esta cohorte retrospectiva fue lo suficientemente prolongado como para evaluar los objetivos finales propuestos. Asimismo, la regla de predicción clínica* obtenida es de fácil aplicación en la práctica clínica diaria. Las variables pronósticas que fueron analizadas ya habían sido descriptas en numerosos trabajos ${ }^{1-4}$, inclusive los autores ya habían reportado su experiencia ${ }^{4-5}$.

Vale aclarar que el verdadero valor de esta estratificación pronóstica deberá ser confirmado cuando se la aplique sobre otras poblaciones con mieloma indolente -cohorte de validación* de la regla de predicción clínica- para ver si mantiene la predicción reportada en este trabajo -cohorte de derivación o desarrollo* de la regla de predicción-.

\section{Conclusión del comentador}

La utilidad de un puntaje que permita conocer cuales son los pacientes con mayor riesgo de evolución a mieloma múltiple o amiloidosis permite implementar un seguimiento mas estrecho, estudiar en esta población los factores relacionados a la biología de la enfermedad que nos permitan entender los mecanismos de progresión, y posiblemente en un futuro, seleccionar e implementar estrategias terapéuticas.

Jorge Arbelbide [ Sección de Hematología. Servicio de Clínica Médica Hospital Italiano de Buenos Aires. Jorge.arbelbide@ hospitalitaliano.org.ar ]

Ver glosario*

\section{$\begin{aligned} \text { Recibido el 26/10/07 y aceptado el 08/01/08. } & \end{aligned}$}

Arbelbide J. Pronóstico del mieloma indolente. Evid. actual. práct. ambul; 11(1): 11, Ene-Feb.2008. Comentado de: Clinical Course and Prognosis of Smoldering (Asymptomatic) Multiple Myeloma. Robert A. Kyle, M.D., Ellen D. Remstein, M.D., Terry M. Therneau. N Engl J Med 2007;Junio21, 356:2582-90. PMID: 17582068. Disponible en URL: http://content.nejm.org/cgi/reprint/356/25/2582.pdf (último acceso 08/01/08).

\section{Referencia}

1. Cesana $\mathrm{C}$, et al. Prognostic factors for malignant transformation in monoclonal gammopathy of undetermined significance and smoldering multiple myeloma. $\mathrm{J}$ Clin Oncol. 2002 Mar 15; 20(6):1625-34

2. Kyle RA, et al. A long-term study of prognosis in monoclonal gammopathy of undetermined significance. N Engl J Med. 2002 Feb 21; 346(8):564-9.

3. Perez-Persona E, et al. New criteria to identify risk of progression in monoclonal gammopathy of uncertain significance and smoldering mutliple myeloma based on multiparameter flow cytometry analysis of bone marrow plasma cells. Blood. 2007 Jun 18; [Epub ahead of print]

4. Bladé J, et al. Smoldering multiple myeloma and monoclonal gammopathy of undetermined significance. Curr Treat Options Oncol. 2006 May;7(3):237-45.

5. Kyle RA, et al.Long-term follow-up of 241 patients with monoclonal gammopathy of undetermined significance: the original Mayo Clinic series 25 years later. Mayo Clin Proc. 2004 Jul; 79 (7):859-66.

6. Kyle RA, et al. A long-term study of prognosis in monoclonal gammopathy of undetermined significance. N Engl J Med. 2002 Feb 21;346 (8):564-9. 